\title{
Assembly Analysis of Interference Fits in Elastic Materials
}

\author{
Kannan Subramanian and Edward P. Morse \\ Structural Integrity Associates, Inc., 11515 Vanstory Drive, \\ Suite 125, Huntersville, NC 28078 \\ ksubramanian@structint.com \\ Center for Precision Metrology, Dept. of ME\&ES, \\ University of North Carolina at Charlotte, \\ Charlotte, NC 28223 \\ emorse@uncc.edu
}

\begin{abstract}
The objective of this work is to provide fast approximations of force/work/energy calculations for designers of assemblies in which interference is required. In this paper, the subjects of interest are assemblies in which there are several sets of features that can influence the final location of the parts, once assembled. The analysis includes a systematic approach in developing an analytical model involving basic laws of equilibrium and more advanced finite element based models to estimate the work required for assembly and the total strain energy in the components after assembly is complete. In this approach, an ideal press-fit type interference assembly is considered initially and solution methodology is developed. A non-symmetric hinge assembly with multiple interferences is analyzed later with the developed approach and the results are compared with the experimental observations. The suitability of the GapSpace assembly analysis method [1] for assemblies in which there is interference between the components is also investigated.
\end{abstract}

\section{Introduction}

Due to the increasing performance requirements, geometric tolerances on machine components are being driven smaller and smaller. In order to avoid excessive 'slop' or free play between components, it may be desirable to have a small, but non-zero, interference between the components. Press-fit is commonly chosen for faster and cheaper assembly of such an interference-type fit, instead of the cumbersome shrinkfit. These assemblies contain several sets of features that can influence the final location of the parts, once assembled. The interference assembly undergoes contact pressure and friction forces at the contact interface which is a common characteristic of any contact problem. Analytical solutions are difficult to find for even simple geometries due to the non-linear conditions resulting from frictional forces at the contact surfaces. With the advent of high performance computers and efficient computational techniques such as finite element method, complicated interference geometries can also be analyzed with a fair degree of accuracy. In this work, the interference problem 
is considered in a broader and general sense by analyzing a simple interference fit and developing approaches for complex interference assemblies. The key feature of this work is to investigate the assembly force, work done, and strain energy of the press-fit assemblies. A commercially available finite element analysis package, ANSYS 11.0 [2], has been used for analyzing the interference assemblies considered in this work. The ultimate objective is to provide tools to assist designers with fast approximations of the parameters (work, force, and energy) mentioned above.

\section{Simple Problem}

An idealized symmetrical interference assembly involving three members is considered (see Figure 1) for the theoretical development and validation purposes. Assuming that the interference is very small, that is, $\delta \ll c$, all the materials in the assembly are the same and follow linear elastic and isotropic properties, and in the absence of any friction, a simplistic analysis from solid mechanics principles described in [3], results in the following displacements for the three members in the assembly,

$$
\begin{aligned}
& \text { change in length of member } 1\left(\delta_{a}\right)=\delta \frac{a}{L}, \\
& \text { change in length of member } 2\left(\delta_{b}\right)=\delta \frac{b}{L},
\end{aligned}
$$

and change in length of member $3\left(\delta_{c}\right)=\delta \frac{c}{L}$, where $L=a+b+c$.
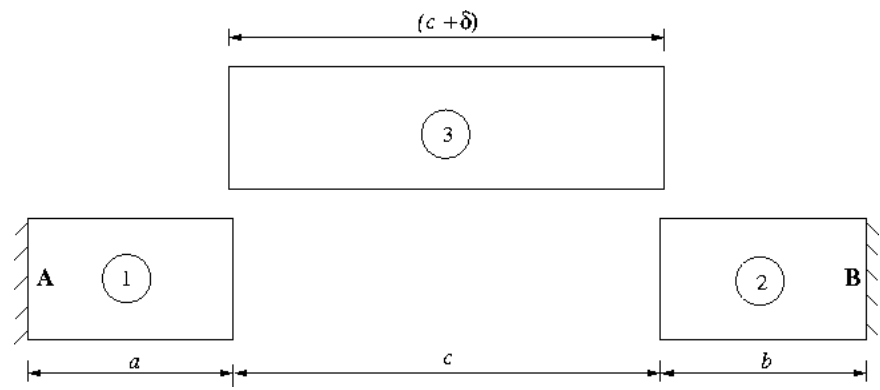

Fig. 1. Idealized interference fitting problem

Due to the symmetry associated with the model, only half of the geometry shown in Figure 2 is modeled in ANSYS with rounded mating corners to overcome numerical instabilities associated with sharp corners. Member 3 is assumed to move incrementally into the space between members 1 and 2 . The contacting surfaces were assumed to be flexible-to-flexible type and 8-noded linear quadrilateral elements are used to mesh the geometry. Plane strain conditions are assumed. The surfaces in contact are assumed to be sliding freely without friction. For a geometry with $a=b=50$ $\mathrm{mm}, \mathrm{c}=100 \mathrm{~mm}$ with an interference of $\delta=1 \mathrm{~mm}$, the displacement results for each member using finite element analysis is found to be in good agreement with the results calculated with the force equilibrium based approach discussed in Reference 3 . 


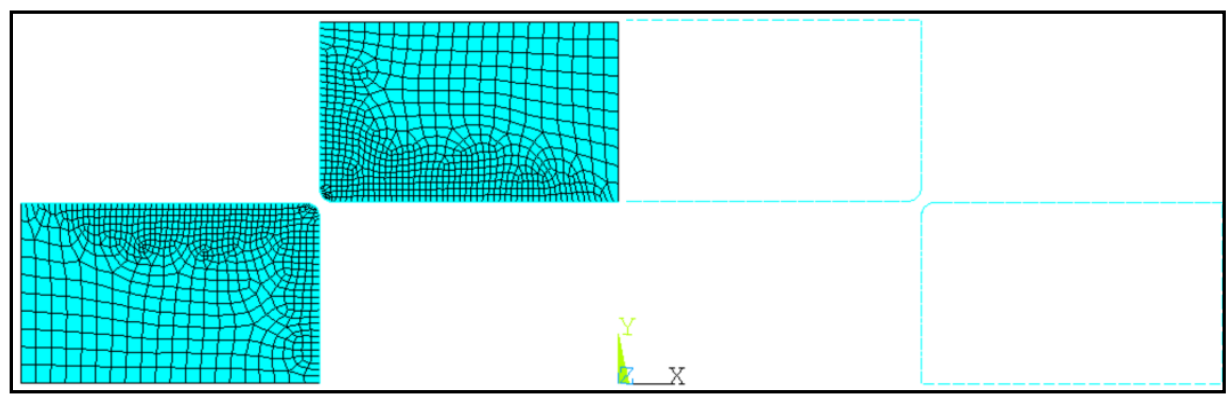

Fig. 2. Finite Element Model for the Simple Problem

That is, a displacement of $0.25 \mathrm{~mm}$ at the end of the assembly is calculated $\left(\delta_{a}=\right.$ $\delta_{c} / 2=0.25 \mathrm{~mm}$ ) irrespective of the material under consideration. In this analysis, low alloy carbon steel material is considered with an Elastic Modulus of $200 \mathrm{GPa}$ and yield strength of $1600 \mathrm{MPa}$. The ANSYS model included a dry friction co-efficient of 0.2 . Displacement contours at the end of the assembly are shown in Figure 3, and it can be observed that the member 1 and 2 are under compression with a maximum displacement of approximately $0.25 \mathrm{~mm}$ which is consistent with the analytical results.

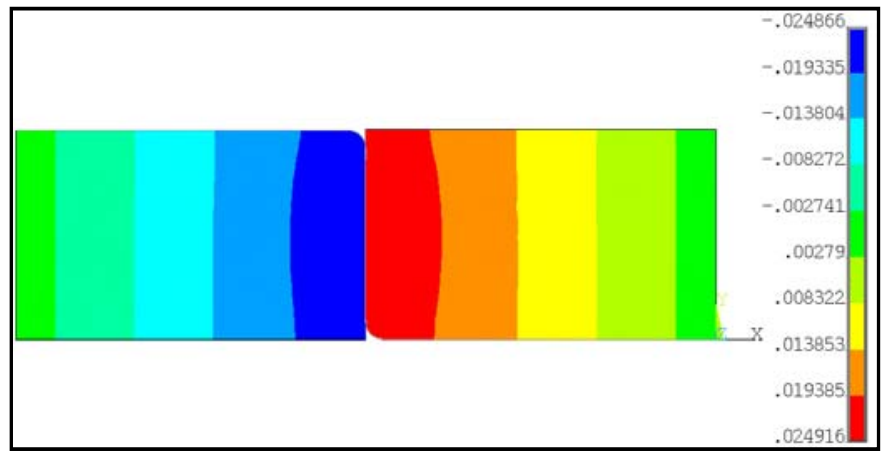

Fig. 3. Displacement (in mm) Results for the Simple Problem

\section{Hinge Assembly}

With the preliminary bench marking described in the previous section, the assembly analysis is extended to a 'hinge assembly' for various combinations of fitting conditions. Figure 4(a) represents such a generic and parametric hinge assembly, Figure 4(b) represents the four GFS pairs in the assembly, and lastly Figure 4(c) characterizes the fitting conditions, and the inequalities needed for non-interfering assembly. The identification of these fits conditions for non-interfering assemblies is described in Reference 1. The extension to this method which is the subject of the current work is simply to relax the requirement that all fits conditions have non-negative values as shown in Figure 4(c). 


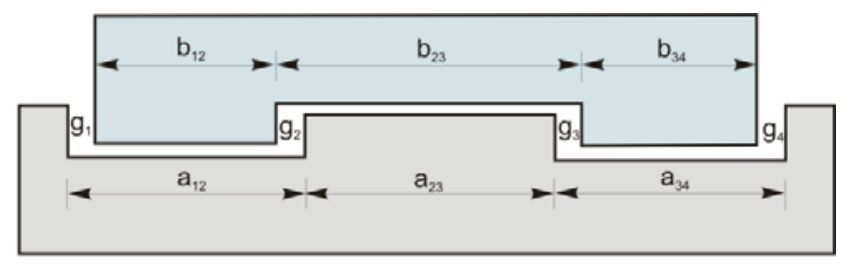

(a) Atwo-part "hinge" assembly

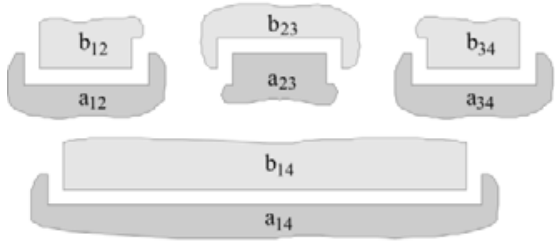

(b) The four GFS pairs in the hinge assembly

$$
\begin{aligned}
& \mathrm{FC}_{1}=g_{1}+g_{2} \geq 0 \\
& \mathrm{FC}_{2}=g_{2}+g_{3} \geq 0 \\
& \mathrm{FC}_{3}=g_{3}+g_{4} \geq 0 \\
& \mathrm{FC}_{4}=g_{1}+g_{4} \geq 0
\end{aligned}
$$

(c) Fits conditions

Fig. 4. General Hinge Assembly with Various Fits Conditions

A series of case studies are considered for the hinge assembly, the first press-fit is one in which, $b_{12}>a_{12}, a_{23}>b_{23}, a_{12}=a_{23}=a_{34}$, and $b_{12}=a_{34}$. In this case, the gaps (negative due to interference) are related in such a way that, $g_{1}+g_{2}=g_{3}+$ $g_{4}$, that is, the assembly is 'symmetric.' Therefore, as in the case of the basic simple geometry discussed for bench marking, only a half model is created in ANSYS and the assembly analysis is carried out. In addition, the sharp mating corners are rounded and interference values are kept low in order to keep the stresses below the yield strength of the subject material. The work done in carrying out the assembly is calculated to be much higher (x8) than total strain energy stored in the final assembled configuration. Comparing the strain energy with the work done, it can be deduced that a large fraction of the work is lost during assembly, likely transformed into heat during the assembly. However, the thermal energy is not calculated for the sake of simplicity. Also, the main focus in this study is on the assembly forces and the work required for such an assembly.

The second and third of the case studies are carried out for non-symmetric cases with differing interferences and are characterized by, $b_{12}>a_{12}, a_{23}>b_{23}, b_{34}>$ $a_{34}, a_{12}=a_{23}=a_{34}$, and $b_{12}>b_{34}$. The gaps are selected in such a way that, $\left|g_{1}+g_{2}\right|>\left|g_{3}+g_{4}\right|$. The total interference in the third case is double that of the second case. Due to the non-symmetry, the whole geometry is modeled in ANSYS and analyzed. In these cases, the calculated stresses are higher in the region of greater interference in line with the physics of the problem (see Figure 5c). The last two cases considered are those in which one feature pair of the assembly "fits perfectly" while other sections have interference. These "just-fit" cases are characterized by $b_{12}>a_{12}$, and $a_{12}=a_{23}=a_{34}$. In addition, for the first just-fit case, $a_{23}=b_{23}$, and $b_{34}>a_{34}$, while for the second just-fit case, $a_{23}>b_{23}$, and $b_{34}=a_{34}$. The gaps are selected in such a way that, $\left|g_{1}+g_{2}\right|>\left|g_{3}+g_{4}\right|$. For the second just-fit case, $g_{3}=g_{4}=0$. Graphically, in the first of the two just-fit cases, the center sections of the parts are of the same undeformed size, while in the second case, the rightmost sections are of the 
same dimension. The stresses and displacement patterns correlate well with the physics of the assembly process. That is, more stresses are observed at the interfering sections while negligible stresses and zero displacements are observed at the just-fit sections.

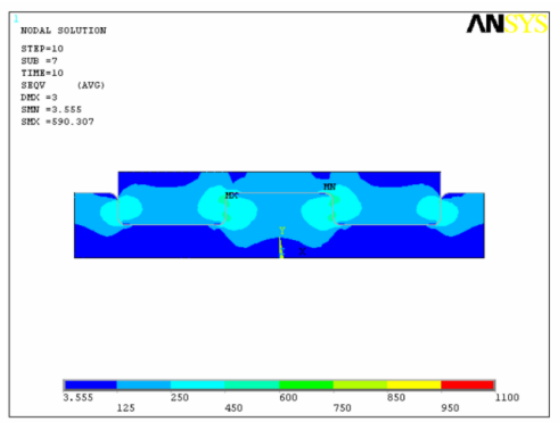

(a) Symmetric Hinge Assembly

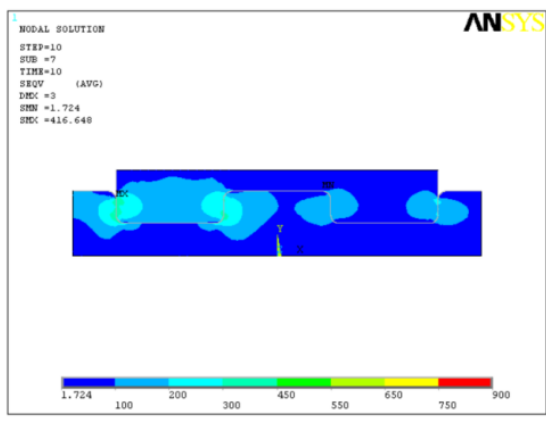

(b) Non-symmetric Hinge Assembly - I

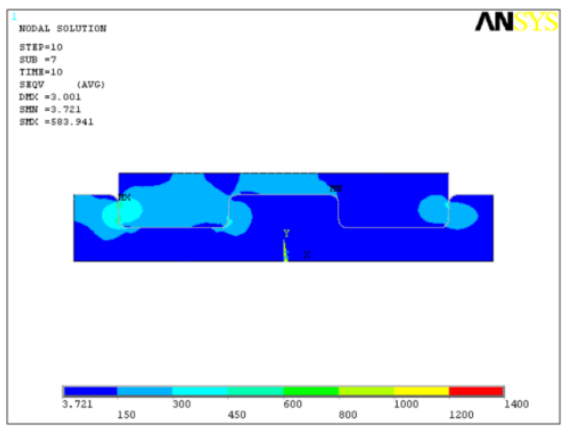

(d) Just-Fit Type Hinge Assembly - 1

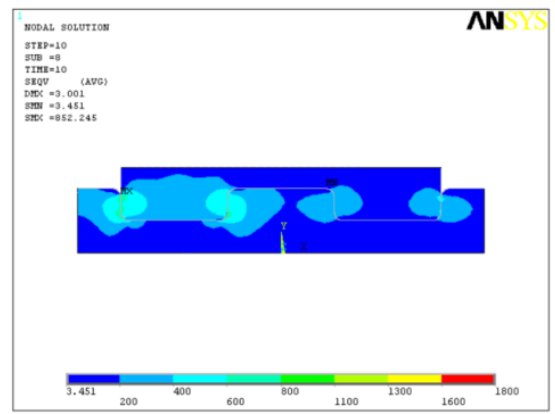

(c) Non-symmetric Hinge Assembly - 2

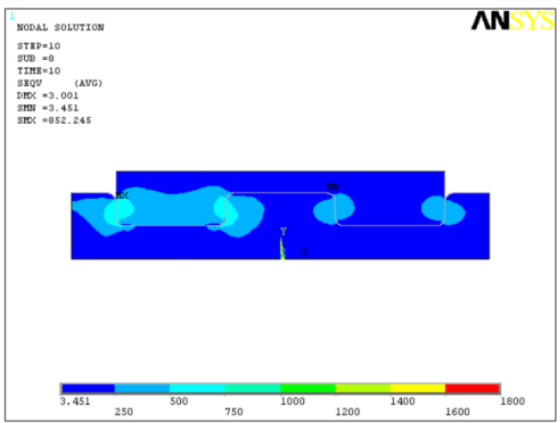

(e) Just-Fit Type Hinge Assembly - 2

Fig. 5. Equivalent Stresses (MPa) in various simulated Hinge Assemblies 
The equivalent or von-Mises stress distribution in the assembled configuration for various hinge assemblies are shown in Figure 5. The boundary conditions imposed for the ANSYS analysis include that the lower part is restrained in the vertical direction and a few nodes at the bottom surface of the specimen are fixed to avoid rigid body motion in the horizontal direction. The top surface of the top member is free to move horizontally (to accommodate the influence of the different interference conditions), and given uniform vertical displacements until it comes to the final assembly position.

\section{Experimental Set-Up}

In an effort to establish the correlation between the numerical results and the forces and stresses observed in actual assembly cases, a series of physical experiments are being developed. The first experimental set-up of this series is shown in Figure 6 . High strength aluminum (2024 with T36 heat treat) material, with yield strength of $325 \mathrm{MPa}$ and Elastic Modulus of $70 \mathrm{GPa}$ is utilized in this study. The aluminum parts are pressed together using the Instron load frame. The parts in assembly are machined in such a way that the assembly will contain multiple interferences with all fits conditions (shown in Figure 2c) being negative. That is, this will be an example of a nonsymmetric case discussed in the previous section and shown graphically in Figure $5 \mathrm{~b}$ and $5 \mathrm{c}$.

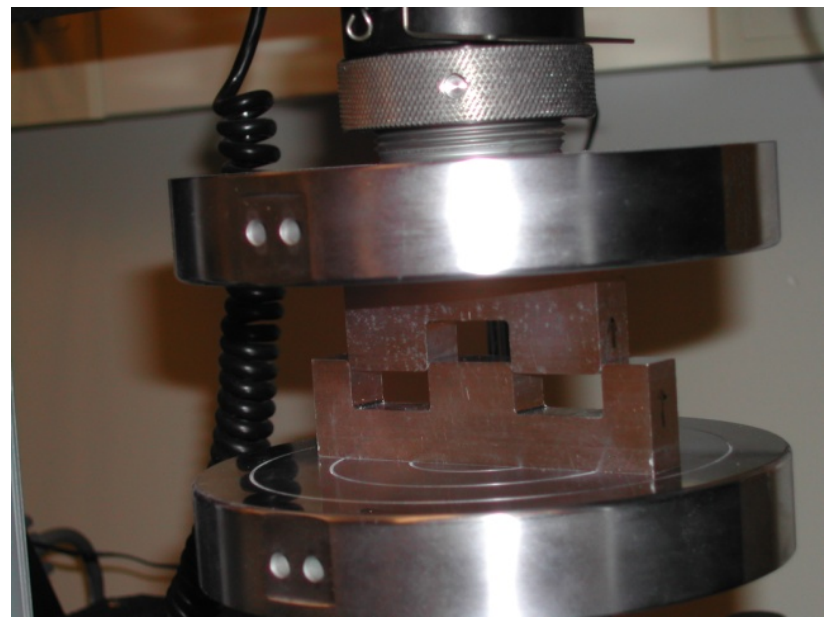

Fig. 6. Experimental Set-up for Hinge Assembly

The specific geometry of the test parts is modeled in ANSYS, and friction is neglected between the mating parts for added simplicity. The equivalent stress at the end of the assembly process is shown in Figure 7. There is some plastic deformation occurring at the filleted regions, and hence the stresses are very high in those areas. However, in the overall bulk geometry of the components, the stresses are well below the yield strength of the material under consideration. 


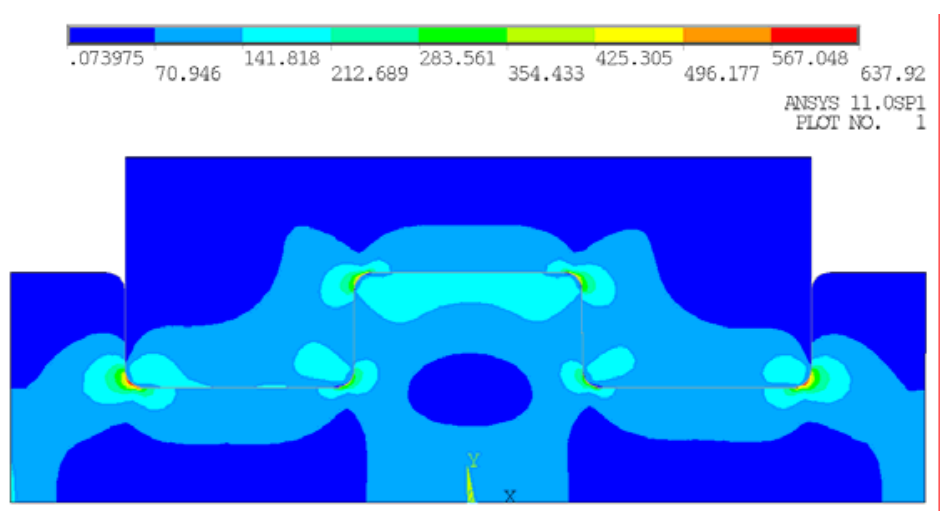

Fig. 7. Equivalent Stress Distribution at the Assembly Condition for Experimental Assembly

\section{Results and Discussion}

The assembly forces were calculated from ANSYS by selecting the top surface and integrating the vertical stress components over the length of the part for each deformation step. Figure 8 shows the comparison of these forces with the Instron-measured compressive forces. The red squares in the graph represent the calculated forces using ANSYS and the solid line represents forces measured during assembly.

During the initial contact, ANSYS predicted very high assembly force value while the measured forces are still lower. This is due to the modeling of the filleted region, and the adjustment of the parts as they become aligned for assembly. The assembly forces reduce as the analysis progresses in ANSYS and eventually begin following the pattern of the experimental observation - a steady increase as more of the parts overlap. The measured forces peaks between 8 and $10 \mathrm{kN}$, while the calculated forces reach $16 \mathrm{kN}$, thus differing by a factor of approximately 1.6. The calculation of the work done during the assembly process is 38.6 Joules based on the measured forces, while the ANSYS calculated work is 104 Joules. The strain energy stored in the assembly is calculated to be 198 Joules. We believe this result is anomalous, and due to the manner in which ANSYS calculates strain energy. The strain energy is higher because the calculation of strain energy includes the elements that are in contact where plastic deformation may occur. However, the work done was calculated only based on the assembly forces and displacements.

The difference in results between the measured and ANSYS calculated work/force/energy can be attributed to several factors, such as contact friction, initial assembly deformation, and how accurately the ANSYS model represented the experimental set-up. The results presented here, while preliminary, are encouraging in that the magnitude of the forces and energy required for assembly are simulated with some agreement. A series of further experiments will help in developing a unified methodology that will predict the assembly forces. 


\section{Force vs. Displacement}

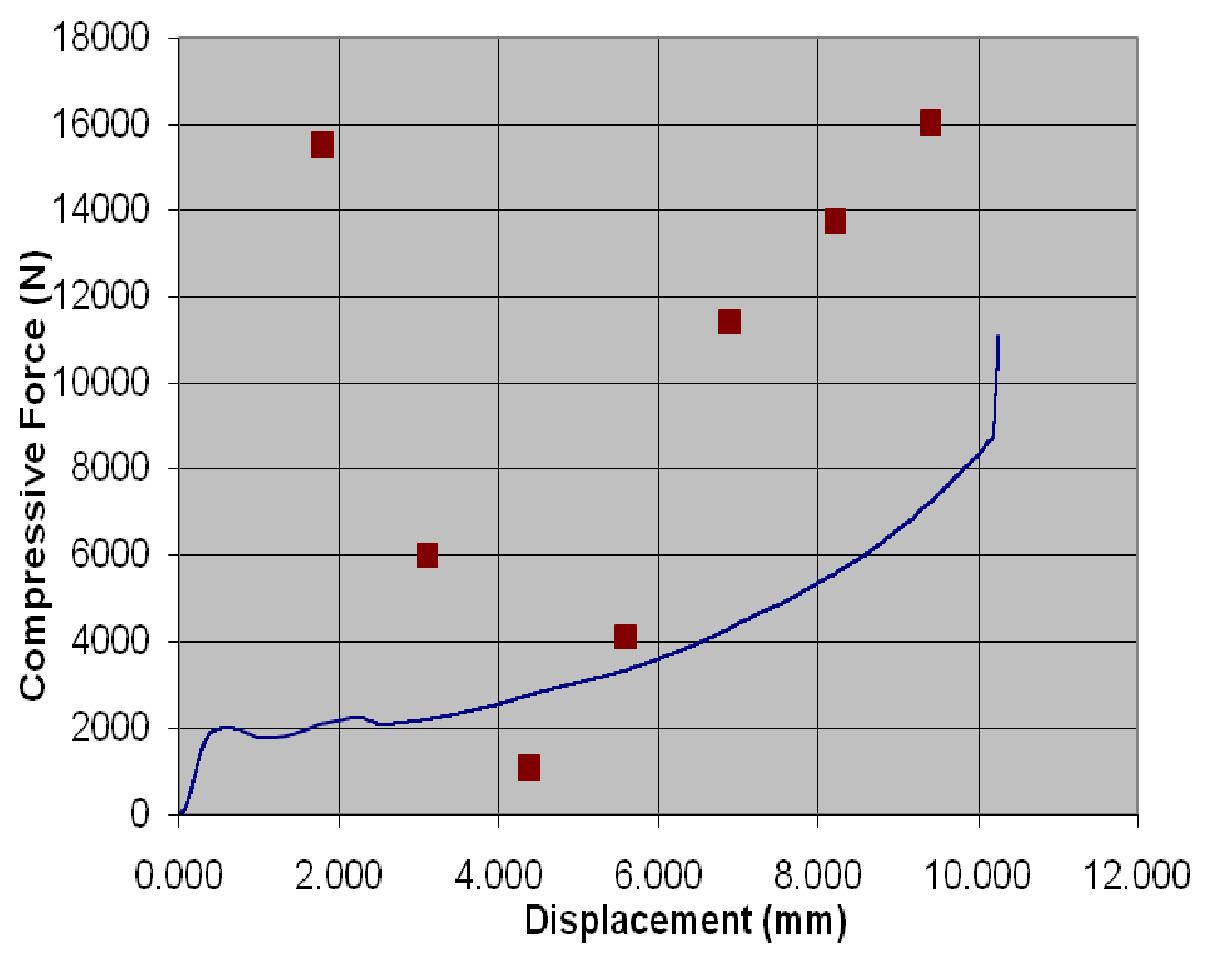

Fig. 8. Comparison between ANSYS and Measured Forces

\section{Conclusions}

It is demonstrated that the final location and displacement of the members of an assembly can be predicted using the identification of fitting conditions and relative starting position of the members. The simplistic cases allows to easily predict the outcome of the analyses, and the continuation of this work will utilize additional numerical experiments to predict the part displacement and location of the members based on the fitting conditions determined using GapSpace techniques. By having a set of test cases that span the variety of interactions possible in the assembly, guidance can be provided to the designer that will allow him or her to more quickly define nominal interference conditions that satisfy design requirements for force, stress, or assembly work. It is clear from the analyses that the interaction of all four of the fits conditions is important to the stress distribution in the components. Additional work will also be required to assess the sensitivity of these design objectives to the dimensions actually obtained in assembly. This will assist in the specification of geometric tolerances on the assembly features, and will be an important next step in assisting the designer. 


\section{Acknowledgments}

The authors acknowledge the support of The University of North Carolina at Charlotte's Center for Precision Metrology, the William States Lee College of Engineering, and the National Science Foundation for its support of initial parts of this work through grant DMI-0237501. Any opinions, findings and conclusions or recommendations expressed in this material are those of the authors and do not necessarily reflect the views of the National Science Foundation. The assistance of Prof. Harish Cherukuri in developing the analytical results is also appreciated.

\section{References}

[1] Zou, Z., Morse, E.P.: A gap-based approach to capture fitting conditions for mechanical assembly. Computer-Aided Design 36(8), 691-700 (2004)

[2] ANSYS/Mechanical, Release 11.0 (w/Service Pack 1), ANSYS Inc. (August 2007)

[3] Subramanian, K., Morse, E.P.: Analysis of interference fits in elastic materials. In: Proceedings of DETC 2005, ASME 2005 Design Engineering Technical Conferences and Computers and Information in Engineering Conference, Long Beach, CA, September 25$28(2005)$ 